\title{
Quentin Meillassoux. Realismo especulativo y Narrativas desde la Contingencia.
}

\section{Quentin Meillassoux. Speculative Realism and Narratives from the Contingency.}

DOI: 10.32870/sincronia.axxv.n80.5b21

\author{
Carlos Alberto Navarro Fuentes \\ Universidad Autónoma de la Ciudad de México (MÉXICO) \\ CE: betoballack@yahoo.com.mx / ID ORCID: 0000-0003-4647-9961
}

\section{Esta obra está bajo una Licencia Creative Commons Atribución-NoComercial 4.0 Internacional}

Recibido: $18 / 02 / 2021$

Revisado: 30/04/2021

Aprobado: 16/06/2021

\section{RESUMEN}

El objetivo de este trabajo es introducir el 'realismo materialista especulativo' de Quentin Meillassoux, estableciendo una postura crítica frente a la tradición metafísica del 'absoluto' que ha prevalecido en la filosofía occidental pos-kantiana, fundamentada en la necesidad de la contingencia que propone. Lo anterior implica realizar una crítica de lo que se ha entendido como realismo, necesidad y existir. Para ello, se van desglosando conceptos claves de la filosofía de Meillassoux ejemplificando su influencia -y posible presencia atrás en el tiempo- sobre otros pensadores y creadores en sus respectivas narrativas como Graham Harman, Timothy Morton, Nick Land y Florian Hecker, quienes ahondan en temas que generan malestar, asombro, nihilismo y pesimismo en las sociedades contemporáneas, como la probabilidad y la predicción en los mercados financieros, el Antropoceno y la naturaleza, la conflictiva relación entre sujeto y objeto, la verdad y el caos, entre otras cosas, sustrayéndonos del discurso humanista sobre el que descansan los paradigmas científicos, financieros y ambientales de nuestro tiempo y que han terminado por resquebrajar la identidad del hombre, siendo la producción capitalista el factor geológico más determinante. 
Reflexionamos sobre los siguientes cuestionamientos. ¿Qué relatos pueden dar cuenta de la condición actual del mundo? ¿Qué relatos surgen cuando dejamos de centrar nuestra atención en el hombre? ¿Qué hábitos de pensamiento nos obliga modificar la conciencia de que todo lo que nos rodea es contingente?

Palabras clave: Materialismo especulativo-Realismo especulativo. Correlacionismo. Contingencia. Pensamiento-Ser. Sujeto-Objeto. Hipercaos.

\section{ABSTRACT}

The objective of this work is to introduce Quentin Meillassoux's 'speculative materialist realism', establishing a critical stance against the metaphysical tradition of the 'absolute' that has prevailed in post-Kantian Western philosophy, based on the need for contingency that he proposes. This implies making a critique of what has been understood as realism, necessity and existing. To do this, key concepts of Meillassoux's philosophy are broken down, exemplifying its influence -and possible presence back in time- on other thinkers and artists in their respective narratives such as Graham Harman, Timothy Morton, Nick Land, and Florian Hecker, who delve into issues that generate discomfort, amazement, nihilism and pessimism in contemporary societies, such as probability and prediction in financial markets, the Anthropocene and nature, the conflictive relationship between subject and object, truth and chaos, between other things, subtracting ourselves from the humanist discourse on which the scientific, financial and environmental paradigms of our time rest and which have ended up cracking the identity of man, with capitalist production being the most determining geological factor. Let us to reflect on the following questions. What narratives can give an account of the current condition of the world? What narratives emerge when we stop focusing our attention on man? What habits of thought force us to change the awareness that everything around us is contingent?

Keywords: Speculative materialism-Speculative realism. Correlationism. Contingency. Thought-Being. Subject-Object. Hyperchaos.

El Quijote es un libro contingente, el Quijote es innecesario.

Puedo premeditar su escritura, puedo escribirlo, sin incurrir en una tautología....Mi recuerdo general del Quijote, simplificado por el olvido y la indiferencia, puede muy bien 
equivaler a la imprecisa imagen anterior de un libro no escrito.

Postulada esa imagen (que nadie en buena ley me puede negar) es indiscutible que mi problema es harto más difícil que el de Cervantes. Mi complaciente precursor no rehusó la colaboración del azar: iba componiendo la obra inmortal un poco à la diable, llevado por inercias del lenguaje y de la invención. "Pierre Menard, autor del Quijote" (Borges, 1987)

\section{Introducción al materialismo especulativo de Quentin Meillassoux}

Después del agotamiento del giro lingüístico que dio la filosofía en el siglo XX, se han venido explorando epistemologías, ontologías, éticas y perspectivas científicas en donde la conciencia humana no es ya el centro de atención. Quentin Meillassoux (1967), compartiendo el interés por las matemáticas con su mentor Alain Badiou, quien introduce la obra del primero Después de la finitud. Ensayo sobre la necesidad de la contingencia (2015a), escrita en 2006 y traducida al castellano en 2015, afirma que las matemáticas alcanzan a las cualidades primarias de las cosas, en oposición a las cualidades secundarias manifestadas en la percepción. Este descubrimiento proviene de la ontología de la teoría de conjuntos del matemático Georg Cantor. ${ }^{1}$ Luego de publicar Meillassoux su

${ }^{1}$ Georg Ferdinand Ludwig Philipp Cantor (1845-1918). En 1873 Cantor probó que los números racionales son numerables, es decir, se pueden poner en correspondencia biunívoca con los números naturales. También probó que los números algebraicos, son numerables. Sin embargo, sus intentos por decidir si los números reales son numerables resultaron más difíciles. En diciembre de 1873 logró probar que el conjunto de los números reales no era numerable y en 1874 lo publicó en un artículo. Es en este artículo que aparece por primera vez la idea de una correspondencia biunívoca, aunque sólo queda implícita en el trabajo. En su trabajo de 1874, Cantor probó que en cierto sentido 'casi todos' los números son trascendentes, al probar que los números reales no son numerables, mientras que los números algebraicos sí lo son. El conjunto de Cantor, llamado así por ser aporte de Georg Cantor1 en 1883, es un destacado subconjunto fractal del intervalo real [0,1]. Además de una curiosidad matemática, contradice una intuición relativa al tamaño de objetos geométricos: es un conjunto de medida nula, pero no es vacío ni numerable. Se construye de modo recursivo dando los siguientes pasos: El primer paso es tomar el intervalo [0,1]. El segundo paso es quitarle su tercio interior, es decir el intervalo abierto $(1 / 3 ; 2 / 3)$. El tercero es quitar a los dos segmentos restantes sus respectivos tercios interiores, es decir los intervalos abiertos $(1 / 9 ; 2 / 9)$ y $(7 / 9 ; 8 / 9)$. Los pasos siguientes son idénticos: quitar el tercio de todos los intervalos que quedan. El proceso no tiene fin. Ver, Georg Cantor, "On the Power of Perfect Sets of Points (De 
obra aludida, ha llamado poderosamente la atención de filósofos, artistas y creadores en general, abriendo un nuevo campo de investigación que desde distintos ámbitos se cuestiona sobre el conocimiento de lo absoluto. En la era moderna -y podemos incluir a la posmoderna- esta ambición habría podido considerarse ingenua de acuerdo con la gnosis realista y la tradición epistemológica en ciernes. Su postura crítica de corte materialista y especulativa tiene un rigor racional que atrae de manera decisiva a muchos pensadores hasta cierto punto frustrados, en gran parte por los límites de las teorías en que la modernidad los encasillo; y en parte, porque la posmodernidad no les dio lo que nunca les prometió.

La transformación de la visión del mundo que nos ofrece es un paso importante para ir más allá de una visión antropocéntrica del Universo. Después de entender su ontología uno no puede más que estar sorprendido por el hecho de que la existencia en la Tierra no esté comandada por ninguna necesidad real ni por ningún Dios; sino que la vida es un 'milagro' de su naturaleza contingente. Para Meillassoux no hay ninguna necesidad que afirmar más que la contingencia: el hecho de que hay devenires y acontecimientos más allá de las probabilidades que lo existente nos permite pensar. Su descubrimiento consiste, a grandes rasgos, en haber observado que en los conjuntos numéricos hay elementos que no pueden ser contados por los modelos matemáticos que lo preceden, abriendo un nuevo campo en el entendimiento del infinito y del absoluto. Retomando un aspecto olvidado del pensamiento de Descartes, pero inyectándolo de conocimientos matemáticos que no existían en su época, Meillassoux nos hace ver que las propiedades primarias de los objetos, es decir aquellas que no dependen del sujeto que las percibe, pueden ser conocidas por medio de los números. El ejemplo que pone es el estudio de la ancestralidad, el cual, gracias al análisis numérico de las propiedades de los minerales, los geofísicos han sido capaces de determinar las eras por las que ha pasado la Tierra, a pesar de que no hay presencia humana que pudiera haber presenciado estos fenómenos.

la puissance des ensembles parfait de points)", en Acta Mathematica 4 (1884), pp.381-392. English translation reprinted in Classics on Fractals, ed. Gerald A. Edgar, Addison-Wesley (1993). 
Se pregunta Meillassoux,

¿De qué hablan los astrofísicos, los geólogos o los paleontólogos cuando discuten la edad del universo, la fecha de formación de la Tierra, la fecha del surgimiento de una especie anterior al hombre, la fecha del surgimiento del hombre mismo? ¿Cómo captar el sentido de un enunciado científico que se refiere explícitamente a un dato del mundo postulado como anterior a la emergencia del pensamiento, e incluso de la vida, es decir, postulado como anterior a toda forma humana de relación con el mundo? (Meillassoux, 2015, p.36).

Muy a su estilo narrativo de indagación-exposición, el filósofo francés prosigue cuestionándose: “¿qué interpretación el correlacionismo es susceptible de dar de los enunciados ancestrales?” (Meillassoux, 2015, p.37). Por ancestral entendemos toda la realidad anterior a toda forma registrada de vida sobre la Tierra. “¿Cómo interpretar un enunciado ancestral?” (Meillassoux, 2015, p.37). En un artículo intitulado "Contingencia y absolutización del Uno", Meillassoux explica:

Por correlacionismo entiendo, en una primera aproximación, a toda filosofía que sostiene la imposibilidad de acceder por medio del pensamiento a un ser independiente del pensamiento. No tenemos nunca acceso, según este tipo de filosofía, a un objeto (entendido en un sentido general) que no esté ya correlacionado a un acto de pensamiento (citado en Ramírez, 2016, p.73).

Si partimos de la perspectiva cartesiana, este sería aquél enunciado cuyos referentes, aunque sean pasados pueden ser planteados como reales ya que gozan de validez por la ciencia experimental en algún momento de su desarrollo. No obstante, "para el correlacionista, semejantes enunciados se esfuman desde el momento en que se actualiza la autocontradicción, según el resplandeciente, de la definición del archifósil: donación de un ser anterior a la donación. 'Donación de un ser'” (Meillassoux, 2015, p.43). Se denomina archifósil o materia fósil "no a los materiales que indican huellas de vida pasada, que son los fósiles en sentido propio, sino a los materiales que indican la existencia de una realidad o de un acontecimiento ancestral, anterior a la vida terrestre" (Meillassoux, 2015, p.37). Por tanto: 
Un enunciado ancestral es verdadero, según el correlacionista, en el hecho de que está fundado en una experiencia presente -realizada sobre un material-fósil dado- y universalizable (verificable en principio por cualquiera). Se puede decir entonces que el enunciado es verdadero porque se apoya en una experiencia en principio reproductible por todos (universalidad del enunciado), sin creer ingenuamente que su verdad provendrá de una adecuación a la realidad efectiva de su referente (un mundo sin donación de mundo) (Meillassoux, 2015, p.45).

La distancia que toma Meillassoux de Descartes incide en el cogito cartesiano, pues el 'cogito correlacional' difiere del primero en al menos dos aspectos:

1) El cogito correlacional no se identifica necesariamente con una metafísica de la representación, porque puede remitir a una concepción de la correlación serpensamiento diferente de la del sujeto y el objeto (como la co-apropiación heideggeriana del ser y el hombre); 2) No se trata de un cogito solipsista en sentido estricto sino más bien de un 'cogitamus', porque funda la verdad objetiva de la ciencia en el acuerdo intersubjetivo entre conciencias. El 'cogito correlacional', sin embargo, instituye también un cierto tipo de solipsismo 'de la especie' o 'de la comunidad': porque consagra la imposibilidad de pensar una realidad anterior, o incluso posterior, a la comunidad de seres pensantes. Esta comunidad solo tiene que vérselas consigo misma y con el mundo que le es contemporáneo (Meillassoux, 2015, pp.87-88).

Además de este concepto de ancestralidad, el filósofo francés introduce algunos otros conceptos que iremos definiendo en el desarrollo de este trabajo, como el de la contingencia, correlacionismo, donación, factualidad, irrazón, entre otros, como parte del núcleo de su crítica 'ante-lo-dado' funcionando como 'lo-que-es' 'por-estar-dado' como universal, absoluto, verdadero y propio de la factualidad. En la obra de Jorge Luis Borges (1899-1986) intitulada Ficciones (1944), encontramos un 
párrafo en el primer relato que lleva el nombre de "Tlön, Uqbar, Orbis Tertius" en el que Borges ilustra -acorde con el idealismo filosófico- un mundo - Tlön- cuyos habitantes conciben lo real como un producto de la mente, de la conciencia. Aquí un extracto que puede funcionar para ilustrar parte del 'espíritu' crítico-reflexivo del materialismo especulativo de Meillassoux:

Los metafísicos de Tlön no buscan la verdad, ni siquiera la verosimilitud: buscan el asombro. Juzgan que la metafísica es una rama de la literatura fantástica. Saben que un sistema no es otra cosa que la subordinación de todos los aspectos del universo a uno cualquiera de ellos. Hasta la frase "todos los aspectos" es rechazable, porque supone la imposible adición del instante presente y de los pretéritos. Tampoco es lícito el plural "los pretéritos", porque supone otra operación imposible... Una de las escuelas de Tlön llega a negar el tiempo: razona que el presente es indefinido, que el futuro no tiene realidad sino como esperanza presente, que el pasado no tiene realidad sino recuerdo presente. ${ }^{2}$ Otra escuela declara que ha transcurrido ya todo el tiempo y que nuestra vida es apenas el recuerdo o reflejo crepuscular, y sin duda falseado y mutilado, de un proceso irrecuperable. Otra, que la historia del universo -y en ellas nuestras vidas y el más tenue detalle de nuestras vidas- es la escritura que produce un dios subalterno para entenderse con un demonio. Otra, que el universo es comparable a esas criptografías en las que no valen todos los símbolos y que sólo es verdad lo que sucede cada trescientas noches. Otra, que mientras dormimos aquí, estamos despiertos en otro lado y que así cada hombre es dos hombres.

Entre las doctrinas de Tlön, ninguna ha merecido tanto escándalo como el materialismo (Borges, 1987, pp.23-24).

Al principio de factualidad, Meillassoux le presenta el abandono del principio de razón por medio del principio crítico-especulativo de irrazón intentando alcanzar un absoluto no metafísico capaz de horadar el círculo correlacional de la razón última. Tal principio implica la necesidad de la contingencia del ente, cuya existencia también es contingente y no una necesidad en el mundo. Si el

\footnotetext{
2 Jorge Luis Borges cita al interior de dicho relato a Bertrand Russell de Analysis of the mind, 1921, p.159, donde se supone que el planeta ha sido creado hace pocos minutos, provisto de una humanidad que "recuerda" un pasado ilusorio.
} 
ente cualquiera que este fuera se tratara de un hecho o de una necesidad, además de negar su contingencialidad en el mundo, confirmaría una facticidad de la facticidad, pudiéndole conceder a este un carácter de absolutización en una secuencia que se extendería lógicamente hasta el infinito. La facticidad de la facticidad se explicaría por leyes no solo universales sino además inmutables y acordes a un universo-dado, lo cual resulta ser contrario a la absolutización óntica especulativa que teoriza Meillassoux en oposición a una hipotético-causal (Hume) que atañe a todos los entes, lo cual implica entre otras cosas como veremos un poco más adelante con Timothy Morton, que la estabilidad de la naturaleza y del planeta pudiera asumirse como un hecho independiente del pensamiento, lo cual guarda relación con el Teorema de Cantor y la 'intotalización de los posibles' (Hotel Kafka) o 'de lo transfinito', en donde no es posible captar en un solo conjunto todos los mundos posibles. De ser posible lo anterior, es decir, que todos los mundos posibles pudiesen ser totalizados en un solo conjunto, hablaríamos de una absolutización ontológica, como sucede con 'todos los aspectos', 'los pretéritos', 'ya todo el tiempo' que señalan 'los metafísicos de Tlön' del relato borgiano.

En los círculos académicos anglosajones se ha venido hablando de 'realismo especulativo'. Pero, 'realismo especulativo' es un término poco exacto que se asigna a perspectivas filosóficas que pueden resultar ser muy distintas entre sí y que tienen en común la tentativa de combatir la postura antirrealista de la fenomenología, el estructuralismo y prácticamente todas las escuelas subsecuentes del pensamiento Occidental, las cuáles piensan que los fenómenos dependen de la mente para existir. La conciencia aquí otra vez. Para efectos de este trabajo, nos referiremos indistintamente a 'realismo especulativo' y 'materialismo especulativo' cuando se trate específicamente de la postura intelectual del pensador francés, para evitar confusiones. Pero ¿cómo acercarnos narrativamente a la exploración de conceptos que trascienden la conciencia humana? Para Meillassoux, el objetivo central del 'materialismo especulativo' como reflexión crítico-filosófica es el descubrimiento de un absoluto, que no es otra cosa que la 'necesidad de la contingencia', lo cual incluye a los entes, siendo la no necesariedad de estos lo único necesario. Por lo que el absoluto solo puede existir no siendo ente, no habiendo sido ni siendo, si no acaso, por venir, por 
ser. De esta manera, lo que se encuentra fuera del pensamiento debe de permanecer impensable. Esta postura la han Ilamado los 'realistas (materialistas) especulativos' -entre ellos Meillassoux-: correlacionismo. Pero, afirma Meillassoux:

Contra el dogmatismo, importa mantener el rechazo de todo absoluto metafísico; pero contra la violencia argumentada de los fanatismos diversos, importa volver a encontrar en el pensamiento un poco de absoluto, el suficiente, en todo caso, para oponerse a las pretensiones de aquellos que se querrían sus depositarios exclusivos, por el solo efecto de alguna revelación (Meillassoux, 2015, p.85).

La crítica contra el dogmatismo es una crítica contra la facticidad conformada de correlaciones que han conducido al extravío del absoluto y la producción de diversas formas de fanatismos e ideologías en ámbitos diversos que van más allá de la religión y la política, como el capitalocentrismo y el antropocentrismo, ambos zócalos geológicos sobre los que descansan el Antropoceno y el Capitaloceno como arenas movedizas que amenazan con engullir por completo la vida en el planeta.

\section{Ficciones más allá de la ciencia}

No resulta desatinado considerar que la postura realista especulativa inició con Kant; o, lo que él Ilamó: revolución copernicana'. No obstante que, algunos filósofos de esta corriente la han llamado 'revolución ptolomeica', ya que el efecto que tuvo fue mantener la percepción humana como centro del pensamiento. Para la mayoría de las filosofías occidentales después de Kant, la cosa-en-sí (noúmeno), es decir, los fenómenos más allá de la percepción humana son impensables. George Harman lo pone en estos términos:

Por un lado, el cientificismo insiste en que la conciencia humana no es nada especial y debe ser naturalizada como el resto de las cosas. Por otro lado, también quiere preservar el 
conocimiento como un tipo especial de relación con el mundo bastante diferente de las relaciones que las gotas de lluvia o lagartos mantienen con el mundo. [...] Pese a todo su regodeo con el hecho de que las personas son trozos de materia como todo lo demás, sostienen que el estatuto mismo de la enunciación es, de algún modo, especial. Para ellos, las gotas de lluvia no saben nada, los lagartos saben muy poco y algunos seres humanos tienen mayor conocimiento que otros. Esto sólo es posible porque se le da al pensamiento una capacidad única para negar y trascender la experiencia inmediata, algo que la materia inanimada es incapaz de hacer, según estas teorías, obviamente. En síntesis, a pesar de sus afirmaciones oscuras sobre la no existencia de lo humano, el cientificismo coloca la estructura del pensamiento en la cima ontológica (Harman, 2011).

Es importante hacer ver que contingencia no es lo mismo que probabilidad y azar. ${ }^{3}$ Las crisis económicas y hecatombes financieras tanto de particulares como de estados a punto de la quiebra en los últimos cien años, nos han llevado a un paréntesis conceptual respecto del funcionamiento de los mercados financieros y la relación riesgo / rendimiento en el tiempo. Ningún modelo de predicción parece poder aplicarse con efectividad y ser considerado racionalmente óptimo. ¿Se trata del fin de la probabilidad? ¿Qué asociación podría tener esto con la 'necesidad de la contingencia' que estipula Meillassoux en Después de la finitud? Elie Ayache (2010) en su obra The Blank Swan. The End of Probability, argumenta que es debido a que el desempeño del mercado en virtud de diversos factores contingentes, unos creados voluntariamente, otros inesperados catastróficos o no, que este suele moverse periódicamente fuera de los intervalos de predicción a los que las matemáticas y las leyes de probabilidad teorizaban y pronosticaban, trazando así vasos comunicantes con la contingencia meillassouxiana y los 'metafísicos de Tlön' del relato borgiano, cuyos resultados nunca se pueden anticipar.

No podríamos afirmar que los mercados se comportan irracionalmente porque hasta ahora al menos, estos no piensan ni bien, ni mal, ni hacen uso de razón; no tienen conciencia del tiempo ni

\footnotetext{
${ }^{3}$ Ver, Elie Ayache, The Blank Swan. The End of Probability, Estados Unidos: Hoboken Wiley, 2010.
} 
del espacio, ni del pasado ni del futuro. En el relato borgiano "Pierre Menard, autor del Quijote", es posible leer “...o a Don Quijote en Wall Street” (Borges, 1987, p.39). Imaginemos que Pierre Menard es un corredor bursátil capaz de replicar a la perfección y sin contratiempos al Quijote de Cervantes, o incluso adelantarse a la escritura de este y hasta a producir algo mejor aún a lo que llegó el nacido en Alcalá de Henares en su sala de inversiones y remates. Esto es, que Menard pudiese predecir en su sala de inversiones el algoritmo de replicación perfecta del Quijote y lograra cotizar su creación en la bolsa de valores. El riesgo en las inversiones como parte de la 'ontología' de los mercados financieros, hijastros del capitalismo en sus versiones hermanas: virtual, electrónica y cibernética conlleva a pensar la contingencia más allá de las probabilidades asignadas por la razón y la ciencia.

Porque en el mismo instante en que se produce ese desvanecerse en el aire, atisbamos por primera vez ese iceberg demasiado sólido entre la niebla. [...] Dudo seriamente si el capitalismo puede llevar adelante la tarea de procesar los hiperobjetos. En algún otro lugar sostuve que como la maquinaria más básica del capitalismo es reactiva más que proactiva, tiene una falla que la hace incapaz de abordar por completo la emergencia ecológica. El capitalismo construye a partir de los objetos en tanto "material en bruto" (lo que sea que entra por la puerta de la fábrica). El estilo retroactivo del capitalismo se refleja en la ideología del "consumidor" y su "demanda" que el capital "encuentra". (Borges, 1987, p.39).

La contingencia -algo que Meillassoux extrae de las matemáticas de Georg Cantor-, es aquello que puede acontecer más allá de lo existente y, por lo tanto, va más allá de las posibilidades dadas. En cierto sentido, la contingencia de Meillassoux es aquello que bien podría justificar las catástrofes antropocénicas incausadas para criticar la noción romántica que los ambientalistas tienen de la naturaleza, tanto como los milagros realizados por dios y otras formas de intervención divina. ${ }^{4}$ Para

\footnotetext{
${ }^{4}$ Ver, Roc Jiménez de Cisneros, "Timothy Morton: una ecología sin naturaleza", en CCCBLAB, Investigación e Innovación en Cultura, entrevista publicada el 13 de diciembre de 2016 en http://lab.cccb.org/es/timothymorton-ecologia-sinnaturaleza/lab (Morton, 2013). Timothy Morton es un filósofo y autor entre otras cosas del concepto "Dark Ecology", que propone repensar la visión de la ecología, el antropocentrismo y el arte. "Timothy Morton es un filósofo y experto en la literatura del período romántico. Su obra, provocadora y extremadamente personal, parte de una compleja matriz de temas y referencias - de Shelley a My Bloody Valentine, del budismo a las cadenas de ADN- a través de las cuales
} 
éste, "el final de la metafísica, al sustraerle a la razón todas sus aspiraciones al absoluto, tomó la forma de un retorno exacerbado de lo religioso" (Meillassoux, 2015, p.78).

La solución de Meillassoux para pensar la cosa-en-sí es el hipercaos. Es este el concepto que Meillassoux nos da como arma para lanzarnos con el pensamiento hacía lo absoluto, pero sin las certezas metafísicas que han gobernado Occidente. ¿Cómo pensar este hipercaos? Uno de los trabajos más interesantes que se han hecho en el campo del arte sobre el concepto de hipercaos lo realizó el músico experimental Florian Hecker (el cual trabaja en el campo de la psicoacústica), cuya propuesta musical no resulta fácil de digerir. ${ }^{5}$ Lo fascinante de esta obra es que se trata de uno de los experimentos más exitosos para crear ilusiones auditivas en el espectador. Sí bien nuestra capacidad para lograrlo es contingente, al menos tenemos la ventaja de saber cómo se podría escuchar. Meillassoux aborda el problema del caos y la consistencia de las leyes naturales en el contexto de la literatura. ¿Qué significado tiene lo anterior para la incipiente 'conciencia ecológica'? Significa que los seres humanos aún no estamos totalmente aprobados éticamente para introducirnos en la 'ecoontología postantropocéntrica' para hacernos responsables y asignar

articula ideas sobre ontología, ecología o estética, entre otras. Junto con Graham Harman, lan Bogost y Levy Bryant, forma parte del núcleo de la $\mathrm{OOO}$, la ontología orientada a objetos, un movimiento filosófico promovido por Harman en 1999 que propone la reinterpretación marcadamente antiantropocéntrica de nuestra relación con el mundo, los objetos y las jerarquías, resultando además en una variante del realismo especulativo. Morton utiliza elementos de cultura popular y un lenguaje lleno de giros poéticos e irónicos para deconstruir con acidez la crisis ecológica derivada del antropoceno, e introduce nuevos conceptos filosóficos, como el que llama hiperobjetos: objetos tan extensos en el tiempo y el espacio que son imposibles de señalar o detectar directamente, como pueden ser la biosfera o el calentamiento global. Lejos de las formas tradicionales de ecocrítica cercanas a la contracultura de finales del XX, la noción de Dark Ecology de Morton es una piedra de toque realista diseñada para romper la cúpula de cristal que todavía nos aísla mentalmente (en un esfuerzo por simplificar y paliar el sentimiento de culpa colectivo) de eso que denominamos entorno. Así, salvando las distancias entre la asimetría, la causalidad, la fenomenología, la coexistencia o la temporalidad, las teorías de Morton subrayan el trauma inherente a nuestra era, con la voluntad de despertar, idealmente, una conciencia que conduzca a una reevaluación del papel y el impacto de la humanidad sobre el planeta. Entre sus libros recientes destacan Realist Magic, Hyperobjects y Dark Ecology" (Morton, 2013).

${ }^{5}$ Ver, Florian Hecker, Octave Chronics https://youtu.be/fkAgEH09r34 (acceso febrero 12, 2021). Aquí se puede leer una conversación entre Florian Hecker y Quentin Meillassoux sobre el concepto de hipercaos: http://www.urbanomic.com/Documents/Documents-1.pdf (acceso febrero 12, 2021). Recomendable recurrir al The Meillassoux Dictionary, Peter Gratton and Paul J. Ennis (ed.) (Edinburgh: Edinburgh University Press, 2015). 
sentido y valor a acontecimientos que suelen justificar su existencia mensurable en términos probabilísticos o estadísticos.

'Ficciones más allá de la ciencia' es un concepto que llama a imaginar mundos donde la ciencia ya no pueda describir los fenómenos que rigen nuestra existencia. En el hipercaos, imaginándolo como un mundo, al ser cualquier cosa posible, efectivamente una de esas posibilidades es la estasis, pero también el cambio incesante, como él trata de imaginar con el concepto de 'fiction without science'. ${ }^{6}$ Los hiperobjetos de Timothy Morton, por citar un ejemplo, son la herramienta 'ecocrítica' que este intelectual y creador designa para deshacer la noción romántica que los ambientalistas tienen de la naturaleza, proponiendo sacar a la 'naturaleza de la ecología' de modo que en parte el ser humano deje de devastar al planeta como resultado de la vida antropocéntrica en la que vivimos-, así como la idea de Heidegger -entre otros- sobre la existencia de un 'mundo' en el que los humanos 'arrojados' en este, no obstante, tuviesen la posibilidad de superar las alienaciones de la modernidad. Y tal vez la mejor manera hacerlo consista en aprender a dejar de ser modernos. Morton considera que

Kant imagina que, aunque estemos limitados en este sentido, nuestras facultades trascendentales están, al menos metafóricamente, flotando en el espacio más allá del borde del Universo, un argumento al que Meillassoux se pliega al sostener que la realidad es finalmente cognoscible exclusivamente por una subjetividad (humana). Y éste es el problema, un problema llamado antropocentrismo (Morton, 2020).

En estos mundos habitados por hiperobjetos, el hipercaos es algo contingente en constante cambio y sin leyes que se puedan definir por medio de la ciencia. Los mundos de los 'metafísicos de Tlön' y de "Pierre Menard, autor del Quijote" son mundos atravesados por hiperobjetos y por el hipercaos, por lo que no es posible asegurar resultados ni hacerse de toda la información ni mediante la razón humana ni la inteligencia artificial alimentada por la antropocéntrica mente humana. Para Morton, es Kant el que muestra -los demás tras de él- que, al inicio mismo del Antropoceno, las cosas nunca

\footnotetext{
${ }^{6}$ Ver, Quentin Meillassoux, Science Fiction and Extro-Science Fiction, Minnesota: Univocal Publishing. (2015b).
} 
o acaso contingentemente coinciden con sus fenómenos, por lo que considera que lo que debe hacerse es extender esta idea revolucionaria más allá de la brecha existente entre el ser humano y el mundo, en donde no haya una sola finitud, sino billones de finitudes, entre ellas cosas, animales, plantas, hombres, mujeres, erupciones volcánicas, tsunamis, etc. De allí la importancia de acabar con esa separación y relación interpuesta entre sujeto y objeto u objeto y sujeto, entre lo que es y cómo aparece. "Lo que el pensamiento ecológico debe hacer, entonces, es enterrar al ser humano, devolviéndolo a la Tierra, es decir, colocarlo en ese objeto gigantesco llamado Tierra dentro de una entidad gigantesca llamada biósfera" (Morton, 2020), lugar en el que por cierto se encontraba en el momento en que se enderezó levantando sus a partir de entonces extremidades superiores.

Por otra parte, ¿qué mecanismos nos pueden arrojar al azar en la realidad? En la música es posible debido a que puede manejarse como un asunto numérico, ¿pero podrá haber mecanismos que hagan lo mismo en el mundo físico? ¿Cuál sería la consecuencia? Reason hace hincapié en la causalidad por lo que algo acontece, en lo cual coincido con Meillassoux en que esto habría que eliminarlo del pensamiento. Por su parte, rationality me parece que tiene una relación más cercana al uso que le da Meillassoux a la ciencia, lo cual es uno de los principales motores de su pensamiento para destruir, precisamente, la razón metafísica.

Leyendo críticamente a Kant, Meillassoux se cuestiona:

¿En nombre de qué, en efecto, podríamos refutar a priori la posibilidad de que no hubiese nada más acá de los fenómenos, y de que nuestro mundo estuviese rodeado por una nada en la cual todo podría finalmente abismarse? Se podría sostener que el fenómeno no está apoyado en ninguna cosa en sí. (Meillassoux, 2015, p.64).

De acuerdo con el materialismo especulativo creeríamos que es efectivamente posible pensar una realidad determinada abstrayendo el hecho que estamos pensando. Este proceder reflexivo sería típico de un materialismo epicureísta, pero de acuerdo a una perspectiva correlacional, no sería posible abstraer de lo real el hecho de un ente 'siempre-ya' dado, pues nada es pensable que no esté 'dado-siempre-ya-a', es decir, el mundo sin ente no es pensable si no ha recibido esta 
donación, es decir, "sin un ente capaz de 'pensar' ese mundo en un sentido general, de intuirlo y de discurrir acerca de él" (Meillassoux, 2015, p.66). A partir de aquí, es importante distinguir entre contingencia y facticidad:

1) La contingencia intramundana que se dice de todo aquello que puede ser o no ser en el mundo, producirse o no producirse en el mundo y esto sin contravenir las invariantes del lenguaje y de la representación a través de las cuales el mundo me está dado; 2) La facticidad de estas invariantes mismas, que remite a la incapacidad esencial en la que me encuentro para establecer su necesidad o su contingencia (Meillassoux, 2015, pp.70-71).

La facticidad, nos ayuda a comprender la 'posibilidad' del Todo-Otro del mundo, y esto en el mismo seno del mundo. Posibilidad ya que estamos en incapacidad para establecer la imposibilidad efectiva del Todo-Otro. "Es impensable que lo impensable sea imposible" (Meillassoux, 2015, p.72). En términos de facticidad esto se traduce en que es racionalmente ilegítimo descalificar un discurso no racional sobre el absoluto con el pretexto de su irracionalidad. Por su parte, la contingencia:

[...] designa la posibilidad, para alguna cosa, de perseverar o de desaparecer indiferentemente, sin que una de esas dos opciones vaya contra las invariantes del mundo. La contingencia designa entonces un saber, el saber que poseo acerca del efectivo carácter perecedero de una cosa determinada. (Meillassoux, 2015, p.92).

Por su parte el caos, de manera general es "ese algo, que el caos nunca puede producir, es un ente necesario. Todo se puede producir, todo puede tener lugar, salvo algo necesario. Porque es la contingencia del ente lo que es necesario, no el ente" (Meillassoux, 2015, p.108).

Es así como Meillassoux crea una filosofía que nos permite ir más allá de lo que se ha llamado el correlacionismo, doctrina filosófica que sostiene la originariedad correlativa entre conciencia y mundo. Forma de pensamiento que ha dominado desde Kant, según el cual solo somos capaces de pensar los fenómenos sensibles. La filosofía de Meillassoux nos invita a pensar por medio de la ciencia fenómenos que se encuentran fuera de la experiencia humana y especular sobre acontecimientos que pueden modificar el contexto de lo que consideramos posible, esto es, 
radicaliza la crítica sobre el círculo correlacional para intentar salir de la correlación concienciamundo de tal modo que permita alcanzar otra condición de verdad que no por haber sido pensada como contradictoria implique necesariamente ser pensada como imposible e imposible pensar sobre ella. Si hacemos nuestra esta concepción del mundo, por lo menos como un ejercicio mental, nos encontramos con uno de los retos más fascinantes a lo que nos arroja la filosofía y la ciencia: transformar nuestros hábitos de pensamiento, significa instalarnos a pensar sobre y en lo de la facticidad.

La reflexión que tal vez debemos hacer sea la siguiente: ¿qué hábitos de pensamiento nos obliga modificar la conciencia de que todo lo que nos rodea es contingente? Adentrarse en la conciencia de la contingencia de las leyes que rigen lo que nos rodea e inclusive de nuestro propio comportamiento, nos permite aceptar lo azaroso del resultado de nuestras acciones. Sin embargo, esa experimentación conlleva a cuestionar aspectos sobre las leyes dentro de lo social y lo artístico. Quizá haya más reglamentaciones en estos campos de lo que generalmente logramos percibir. Para Meillasoux, lo contingente no significa que todo está en constante movimiento, sino que, cuando existe un cambio en el orden aparente, nos damos cuenta que llegamos a otro tipo de orden y no al 'caos'. Lo contingente también puede ser estático por momentos. Por ello tal vez para llegar a un verdadero caos se debe no pensar en los elementos que están dentro del caos -como sería el caso de los hiperobjetos de Morton- sino, en los mecanismos que generan ese caos. Una indeterminación de la indeterminación, llevar al azar el azar. Morton considera que:

Porque en el mismo instante en que se produce ese desvanecerse en el aire, atisbamos por primera vez ese iceberg demasiado sólido entre la niebla. [...] Dudo seriamente si el capitalismo puede llevar adelante la tarea de procesar los hiperobjetos. En algún otro lugar sostuve que como la maquinaria más básica del capitalismo es reactiva más que proactiva, tiene una falla que la hace incapaz de abordar por completo la emergencia ecológica [...] El capitalismo construye a partir de los objetos en tanto "material en bruto" (lo que sea que entra por la puerta de la fábrica). El estilo retroactivo del capitalismo se refleja en la ideología del "consumidor" y su "demanda" que el capital "encuentra" (Morton, 2020, p.121). 
Para Meillasoux, lo contingente no significa que todo está en constante movimiento, sino que cuando existe un cambio en el orden aparente nos damos cuenta de que hemos arribado o nos encontramos en otro tipo de orden y no al caos. Los invito a revisar su ensayo publicado en 2012 "Iteration, Reiteration, Repetition: A Speculative Analysis of the Meaningless Sign", donde especula sobre la posibilidad de que existan signos sin ninguna clase de significado. Aunque sus conclusiones son ambiguas, lo que queda claro es que para crear un signo sin significado es necesario acceder a la eternidad de la contingencia. ${ }^{7}$ Lo que el propio Meillassoux duda es que si un signo sin significado pueda ser suficiente para tener acceso por medio del pensamiento a lo contingente.

En la propuesta de Meillassoux se encuentra en juego básicamente, la relación del pensamiento con el absoluto. Para éste, “[...] El sentido de la desabsolutización: el pensamiento no demuestra ya a priori la verdad de un determinado contenido de piedad, sino que establece el derecho igual y exclusivo de una piedad cualquiera para apuntar a la verdad última" (Meillassoux, 2015, p.82). Para ello será medular comprender el concepto de correlación, ya que a través de éste podemos acceder a la correlación entre ser y pensamiento, pero nunca de manera aislada a uno de ellos, dejando fuera al otro, por lo que el correlacionismo como corriente de pensamiento sostiene el carácter insuperable de la correlación como aquí se ha expuesto, a diferencia de cualquier realismo ingenuo. Afirma Meillassoux que:

El correlacionismo consiste en descalificar toda pretensión de considerar las esferas de la subjetividad y de la objetividad independientemente una de la otra. No sólo hay que decir que nunca aprehendemos un objeto 'en sí', aislado de su relación con el sujeto, sino que debemos sostener también que nunca aprehendemos un sujeto que no esté siempre-ya en relación con un objeto [...] (Meillassoux, 2015, p.29).

Por lo anterior, es importante considerar que la correlación pensamiento-ser no puede reducirse a la correlación sujeto-objeto. Asimismo, no perder de vista que el correlacionismo

\footnotetext{
7 Ver, Quentin Miellassoux, "Iteration, Reiteration, Repetition: A Speculative Analysis of the Meaningless Sign" Freie Universität Berlín, 20 abril 2012 en file:///C:/Users/Alberto/Downloads/quentin-meillassoux-iteration-reiterationrepetition-a-speculative-analysis-of-the-meaningless-sign\%20(1).pdf (acceso febrero 17, 2021).
} 
No sostiene ninguna posición a-racional, religiosa o poética: no pronuncia ningún discurso positivo sobre el absoluto, se contenta con pensar los límites del pensamiento, al ser ellos para el lenguaje como una frontera de las que no se captaría más que un solo borde. El correlacionismo no funda positivamente una creencia religiosa determinada, sino que socava efectivamente toda pretensión de la razón para deslegitimar una creencia en nombre de lo impensable de su contenido (Meillassoux, 2015, pp.72-73).

\section{Conclusiones}

La filosofía de Quentin Meillassoux, materialista especulativa y racionalista crítica, nos ofrece una perspectiva filosófica y crítica que viene a refrescar la tradición del pensamiento -al menos- en Occidente, no solo en Europa. Hoy que los estudios del lenguaje -el giro lingüístico que mencionamos-, la semiótica, la filosofía de la ciencia, la filosofía analítica, la 'revolución' cognitiva y las neurociencias, la biotecnología y los estudios sobre el Antropoceno, entre otras cosas, parecen dominar la atención de gran cantidad de investigadores en el mundo, aunado lo anterior, a la financiarización a la que el tecnocapitalismo ha conducido la economía globalmente y la racionalización-optimización matematizante de la naturaleza, concederle un espacio de oportunidad a la perspectiva filosófica de este pensador de origen francés, resulta además de un reto -por la puesta entre paréntesis de toda o gran parte de la tradición filosófica occidental-, una oportunidad en tiempos de oscuridad e incertidumbre en pleno siglo XXI para repensarnos como humanidad -y no como Humanidad-, más allá de la Modernidad, la posmodernidad, la Ciencia y la Naturaleza como 'formas' ejemplares de la absolutización ontológica y por tanto, de la Necesidad como nos expone Meillassoux en su obra, en particular en Después de la finitud.

La crítica de la crítica que presenta Meillassoux, respecto a las diversas reglas o leyes que nos expone, puede parecer aún y con razón, uno de los puntos todavía por desarrollar o débiles de su pensamiento, ya que no ofrece ejemplos claros y contundentes sobre, por ejemplo, la posibilidad de que las leyes físicas del universo de pronto pudieran ser otras en nuestro mundo. Siempre hemos creído que darnos nuestras propias reglas al momento de crear potencializa nuestra 
capacidad creativa; o los polémicos conceptos tales como un 'Dios virtual' o 'creación ex nihilo'. La anarquía rara vez, para bien y para mal, resulta productiva. Quedan varios cabos sueltos aun por desarrollar de su pensamiento filosófico por parte del filósofo francés, pero estos nadan de momento en la contingencia, son entes que podrían advenir o no, porque estos aun ya habiendo sido pensados o no por él o por alguien más, no son necesarios. Sus reflexiones, no obstante, deben sernos útiles para pensar la ontología, la epistemología, la ética, la política, la fe religiosa, nuestra relación con el medio ambiente y otras formas de vida en el planeta, con el trabajo científico, con la investigación y sus diversas metodologías y métodos, los fanatismos, entre muchas otras cosas, pero tal vez y, sobre todo, la metafísica y el absoluto. No podemos permitirnos más ingenuidad, más cuando nos autodenominamos frente a otros: realistas.

La contingencia como la expone en sus trabajos Meillassoux y los otros trabajos de artistas y creadores que aquí se presentaron, independientemente de los andamiajes teórico-conceptuales de los cuales partan: marxismo, postestructuralismo, etc., presentan reflexiones ético-políticas, obras y concepciones estéticas de carácter crítico y radical que siguiendo o renegando de la filosofía meillassouxiana se insertan en la contingencia o la contingencialidad como potencia del pensamiento en la búsqueda de otro absoluto, de la esperanza (no teológico-religiosa y falsamente esperanzadora) en medio de la oscuridad, el nihilismo, la pérdida de la fe (en todo sentido y respecto de cualquier posibilidad de existencia de absoluto) y el devenir (todavía-no) siempre mejor que prometieron fallidamente primero la Ilustración y luego la Modernidad. Entre estos actores, la tecnología es un elemento en común que emplean para significar y dar sentido a sus obras, siendo esta, otro ente, otro contingente, otra potencia. Refiriéndose a Meillassoux comenta el crítico Terry Eagleton, "mientras hay contingencia, hay esperanza" (Eagleton, 2016, p.15).

\section{Referencias}

Ayache, E. (2010), The Blank Swan. The End of Probability, Estados Unidos: Hoboken Wiley. Borges, J. (1987), Ficciones, México: Emece mexicana. 
Cantor, G. (1884), “On the Power of Perfect Sets of Points (De la puissance des ensembles parfait de points)", en Acta Mathematica 4, pp.381-392. English translation reprinted in Classics on Fractals, ed. Gerald A. Edgar, Addison-Wesley (1993).

Eagleton, T. (2016), Esperanza sin ilusión, Madrid: Taurus.

Gratton, P. \& Ennis, P. (2015), The Meillassoux Dictionary, Edinburgh: Edinburgh University Press.

Harman, G. "Critical Animal with A Fun Little Post", en Object- Oriented Philosophy [blog], 17 de octubre de 2011. http://www.criticalanimal.com/2009/07/

Hecker, F. (9 de septiembre de 2013) Octave Chronics (Conversación entre Florian Hecker y Quentin Meillassoux) [Arhivo de video] https://youtu.be/fkAgEH09r34

Hecker, F. (20 de julio de 2010). This conversation took place during the development of Florian Hecker's piece Speculative Solution, an Urbanomic commission that explored Quentin Meillassoux's concept of 'Hyperchaos'. Speculative Solution: Quentin Meillassoux and Florian Hecker Talk Hyperchaos http://www.urbanomic.com/Documents/Documents-1.pdf Jiménez, R. (13 de diciembre de 2016), “Timothy Morton: una ecología sin naturaleza”, en CCCBLAB, Investigación e Innovación en Cultura, https://lab.cccb.org/es/timothy-morton-ecologia-sinnaturaleza/

Meillassoux, Q. (2015a), Después de la finitud. Ensayo sobre la necesidad de la contingencia, Buenos Aires: Caja Negra Editora.

Meillassoux, Q. (2015b), Science Fiction and Extro-Science Fiction, Minnesota: Univocal Publishing.

Morton, T. (2020), "Hiperobjetos (Fragmento). Emergencia climática”, en Revista de la Universidad de México. UNAM. https://www.revistadelauniversidad.mx/articles/4598b892-bf9d-4c57bdb0-002031bb75fa/hiperobjetos

Morton, T. (2013), Hyperobjects: Philosophy and Ecology after the End of the World, Minnesota: University of Minnesota Press.

Ramírez, M. (2016), El nuevo realismo. La filosofía del siglo XXI, Ciudad de México: UMSNH-Siglo XXI. 